\title{
Does litter size affect emotionality, spatial learning and memory in piglets?
}

\author{
Lisa Fijn $^{\mathrm{a}}$, Alexandra Antonides ${ }^{\mathrm{a}, \mathrm{b}, *}$, Dave Aalderink ${ }^{\mathrm{c}}$, Rebecca E. Nordquist ${ }^{\mathrm{a}, \mathrm{b}}$, \\ Franz Josef van der Staay a, b \\ a Behaviour and Welfare Group, Department of Farm Animal Health, Faculty of Veterinary Medicine, Utrecht University, P.0. Box 80151, 3508 TD Utrecht, \\ The Netherlands \\ ${ }^{\mathrm{b}}$ Brain Center Rudolf Magnus, Utrecht University, Universiteitsweg 100, 3584 CG Utrecht, The Netherlands \\ ${ }^{c}$ Life Sciences, Hogeschool Utrecht, Heidelberglaan 7, 3584 CS Utrecht, The Netherlands
}

\section{A R T I C L E I N F O}

\section{Article history:}

Received 6 November 2015

Received in revised form 28 January 2016

Accepted 28 February 2016

Available online 7 March 2016

\section{Keywords:}

Litter size

Pig

Open field

Cognitive holeboard

Breed

Supplier

\begin{abstract}
A B S T R A C T
Average litter size has steadily increased over the past decades in the pig farming industry. Large litters are associated with an increase of piglets born with a lower birth weight and reduced overall piglet viability. The aim of our study was to investigate whether litter size affects emotionality, learning and memory in pigs. Ten piglets from large litters ( $>18$ piglets) were compared with ten piglets from small litters $(<13$ piglets). Piglets from two different suppliers, using different breeds, (hereafter called: Source) were tested. Effects were determined of Litter size and Source on birth weights and growth rates, on emotionality of the piglets measured in an open field test (OFT) at 5 weeks of age, and on effects of OFT-induced stress as indicated by salivary cortisol. The effects of Litter size and Source on spatial learning and memory in a holeboard task were assessed between 9 and 14 weeks of age. Small litter piglets from Source 1 grew faster than large litter piglets from the same source. This effect of Litter size was not found in piglets from Source 2. In the OFT, no effects of Litter size on behaviours were found. However, piglets from Source 1 had lower baseline cortisol levels, made more escape attempts and showed higher locomotor activity during the OFT than piglets from Source 2. During the acquisition phase of the holeboard task, piglets from Source 2 learned the reference memory component faster and reached a higher overall working memory level in the reversal phase than piglets from Source 1. Our results show that Source (i.e. supplier and/or breed) influenced performance in behavioural tasks, and that the occurrence of litter size effects was supplier or breed dependent.
\end{abstract}

(c) 2016 Elsevier B.V. All rights reserved.

\section{Introduction}

Over the last few decades, high production levels of domesticated pigs with regard to growth and reproduction have been established through genetic selection, the control of reproductive cycles and improvements in husbandry management (Prunier et al., 2010). This has caused a considerable increase in the average litter size of pigs, especially in countries with a large pig industry such as The Netherlands, Denmark, France and Germany (Rutherford et al., 2011). Large litter sizes may affect the welfare of both the sow and her piglets (Rutherford et al., 2013). For example, large litters are

\footnotetext{
* Corresponding author at: Behaviour and Welfare group (formerly Emotion and Cognition Group), Department of Farm Animal Health, Faculty of Veterinary Medicine, Utrecht University, P.O. Box 80151, 3508 TD, The Netherlands.

E-mail addresses: a.antonides@uu.nl, s.antonides@gmail.com (A. Antonides).
}

associated with increased discomfort for the sow during farrowing and an increase in sow teat damage (Norring et al., 2006; Mainau et al., 2010). Sows have a limited uterine capacity, therefore large litters cause intra-uterine crowding (IUC). The uterine circulation increases with a larger number of foetuses, but not proportionally. The limited extent to which the blood flow of the uterus can be increased, results in a decrease of blood flow, oxygen and nutrient availability per foetus (Père and Etienne, 2000). IUC causes competition for resources between foetuses and has detrimental effects on placental development, resulting in increased pre- and neonatal mortality and reduced overall piglet viability (Wahner and Fisher, 2005).

Litter size correlates negatively with size and weight at birth, and birth weight variability within large litters is greater compared to that in small litters (Quiniou et al., 2002; Beaulieu et al., 2010). Litter size also shows a negative correlation with pre-weaning weight gain (de Passillé and Rushen, 1989). Moreover, the risk of 
crushing by the sow is higher in piglets from large litters, as they are generally weaker at birth. Due to their smaller average size, they also have poorer thermoregulatory abilities, and possibly a reduced colostrum intake. Thus, large litter piglets have decreased vitality and therefore an increased risk to die before weaning (Herpin et al., 2002; Rutherford et al., 2013).

Human babies that are born at term but small for gestational age (SGA) or have experienced foetal growth restriction, show poorer neurodevelopmental outcomes than babies that are appropriate for gestational age (Arcangeli et al., 2012). A follow-up study of SGA children showed that they had poorer school performance at adolescence than controls (Larroque et al., 2001). The authors argue that "foetal adaptation to conditions that retard growth during gestation may not be successful in maintaining brain development". Similarly, SGA and foetal growth retardation in rats have negative effects on postnatal growth, metabolism, neurological development and learning ability (Ogata et al., 1985; Saito et al., 2009).

Studies on pigs that are born with a low birth weight (LBW) have been inconclusive. Gieling et al. (2012) found that LBW piglets had higher working memory scores than NBW piglets at the start of the reversal phase of a holeboard task. In a follow-up study, LBW piglets selected with stricter criteria showed improved reference memory performance in both the acquisition and the reversal phase of the same holeboard task (Antonides et al., 2015), warranting further studies. It is conceivable that all piglets from large litters suffer from IUC, as they deal with greater competition over oxygen, space and nutrients than piglets from small litters. Therefore, normal birth weight (NBW) piglets from large litters may have undergone more limitations during foetal (cognitive) development than NBW piglets from small litters.

In the present study, we assessed emotionality and learning ability in ten NBW piglets from large litters and ten NBW piglets from small litters. Of each litter size category, five piglets originated from one supplier, and five from another supplier. We exposed all piglets to an open field test (OFT), in which behaviours such as activity and vocalizations can be used as measures of emotionality (Donald et al., 2011). We then assessed longer-term effects of litter size on cognitive development using the spatial cognitive holeboard task for pigs (Gieling et al., 2012; Antonides et al., 2015).

We expected that piglets from large litters would display more emotional reactivity during the OFT, as expressed by more locomotion, vocalizations and defecations, more suspicion towards a novel object, and less time spent in the centre of the OFT than piglets from small litters. Additionally, we expected large litter piglets to show a greater surge in cortisol after the OFT than small litter piglets. In the holeboard task, we expected large litter piglets to show lower memory scores and longer trial durations and latencies than piglets from small litters.

\section{Material and methods}

\subsection{Ethical note}

This study was reviewed and approved by the animal ethics committee (DEC) of Utrecht University, The Netherlands. The study was conducted in accordance with the recommendations of the EU directive 86/609/EEC. All efforts were made to minimize the number of animals used and to avoid their suffering.

\subsection{Animals}

Based on the information of litter sizes per supplier over the 6 months prior to selection, we determined the upper and lower 25th percentile of these data, resulting in a selection criterion of 13 or less piglets for small litters, and 18 or more piglets for large litters. Because in only a few litters less than 13 piglets were born, the animals were ordered as two separate batches from a pig breeding farm, hereafter called Source 1 . We obtained 10 piglets (T40 $\times$ Pietrain), five from each litter size category (small or large). Unfortunately, due to technical problems it was impossible to obtain the second ordered batch from the same source. Instead, a second batch of 10 piglets (Large White $\times 426$ PIC), again five from each litter size category, was supplied by another pig breeding farm, hereafter called Source 2 . Source 2 supplied piglets bred and reared under SPF conditions. Note that the effects of supplier are indistinguishable from effects of breed. The experimental design hereby changed from a simple test of the effects of litter size (small vs. large) to a two-factorial design with the factors Litter size and Source.

However, for answering our main question whether litter size affects emotionality, spatial learning and memory in piglets, we still had 10 piglets from small litters and 10 piglets from large litters at our disposal. If effects of Litter size are robust, then Source should not be relevant. At the same time, this design enables us to assess the effects of the additional factor Source and its interaction with Litter size. It adds a second question, namely whether effects of Litter size (if present) is robust, i.e. whether or not it is affected by Source (see also Festing et al., 1998; Shaw et al., 2002).

One piglet per litter from a total of 20 litters was selected within $24 \mathrm{~h}$ after birth. All piglets were born to multiparous sows. All piglets of each litter were weighed, including stillborn piglets and piglets that died shortly after birth. The male piglet closest to the average birth weight within its litter was selected and given a different colour ear tag. The male piglet second closest to the average weight of the litter was also marked, in case the selected piglet died before weaning. After the selection process, the piglets remained at the pig breeding farm until weaning.

\subsection{Housing}

At weaning, when the piglets were approximately four weeks old, they were transported to the research facilities of the commercial pig breeding farm of the Faculty of Veterinary Medicine, Utrecht University, The Netherlands. After arrival at the testing facility, all animals were housed under non-SPF (conventional) conditions. The four groups of five piglets were housed in adjacent pens by litter size category (small or large litter) and supplier (Source 1 or Source 2 ). The pens ( $4 \mathrm{~m} \times 5 \mathrm{~m}$ ) were enriched with straw bedding, rubber balls, a gunnysack and chewing sticks. Ambient temperature in the stable was measured daily and ranged between $4{ }^{\circ} \mathrm{C}$ to $27^{\circ} \mathrm{C}$ for the piglets from Source 1 (March-May 2014), and between $8^{\circ} \mathrm{C}$ and $34^{\circ} \mathrm{C}$ for the piglets from Source 2 (April-July 2014). Each pen contained a wooden nest box ( $3 \mathrm{~m} \times 1.5 \mathrm{~m})$ with plastic flaps along the front. Rubber mats, covered with a thick layer of sawdust and straw were placed on the concrete floor of the nest boxes. A heat lamp warmed the nest box until the piglets were 8 weeks of age. The stable in which the pens were located was naturally lighted and ventilated (unheated). Fluorescent lights in the stable were on from $7: 30 \mathrm{~h}$ to $16: 30 \mathrm{~h}$.

To facilitate individual recognition, the animals were marked with a sprayed letter on their back (Porcimark marking spray, Kruuse, Denmark). Food and water were available ad libitum, except during the 5 weeks of holeboard testing, when the animals were fed a quarter of their daily required amount of food in the morning before testing, half of the amount after testing and the remainder in the late afternoon. This feeding schedule ensured that the piglets would not feel saturated during testing and would be motivated to search for food rewards in the holeboard. Starting in the second week after arrival at the research facility, a radio was playing con- 

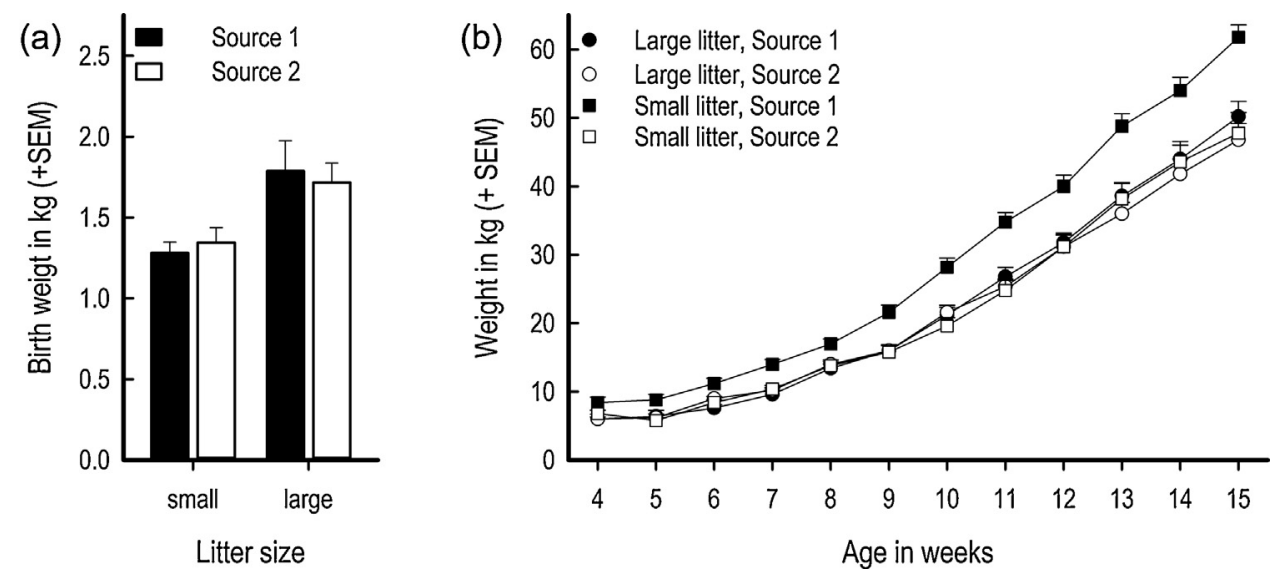

Fig. 1. Births weights (a) and growth (b) of piglets from small and large litters, depicted by source, from weaning at 4 weeks of age until 15 weeks of age.

tinuously at a moderate volume - slightly louder during daytime than at night - to mask noises that could distract or startle the pigs.

\subsection{Birth weight and growth}

The piglets were weighed on the day they were selected (birth weight), on the day they arrived at the research stables (4 weeks old), and then once per week until behavioural testing ended at the age of 15 weeks.

\subsection{Open field test}

\subsubsection{Open field apparatus}

The arena used for the open field test (OFT) measured $2.5 \mathrm{~m} \times 2.5 \mathrm{~m}$ with synthetic walls of $1.5 \mathrm{~m}$ high and was built adjacent to the pigs' home pens by Ossendrijver B.V. (Achterveld, The Netherlands). The arena had one entry door and the concrete floor was covered with a thin layer of sawdust. A camera (JVC Adixxion GC-XA1) recorded continuously during testing hours, and was hung directly above the arena in such a manner that it covered the entire open field arena. The arena was divided into nine equal segments on-screen: one centre segment and eight outer segments (four corner and four wall segments).

\subsubsection{Open field testing}

The OFT was conducted 1 week after arrival at the research facility, when the piglets were approximately 5 weeks old. The piglets had never before been socially isolated. They were taken from their home pen and led through a small corridor into the open field arena, where they remained for $10 \mathrm{~min}$. After $5 \mathrm{~min}$, the experimenter that was standing next to the open field arena manually dropped a novel object (a colourful tambourine toy) in the centre segment of the arena. During the OFT, four behavioural measures of activity were registered manually, using a score form by an observer who stood next to the arena: the number of escape attempts, defined by jumping up against the wall with all four legs off the floor; the total number of defecations; the total number of times the piglet looked at the novel object; and the total number of times the piglet touched the novel object with its snout. During the OFT, eye contact with the piglet was avoided. From the video recordings we scored these behaviours again, as well as: the total number of vocalizations; the total number of line crossings (placing two or more legs in the segment during movement from another segment); and the total time spent in each type of segment (centre or outer).

\subsection{Salivary cortisol}

Two saliva samples were collected per piglet: the first $5 \mathrm{~min}$ before and the second $20 \mathrm{~min}$ after the OFT. The second sample was taken around the expected peak of increased cortisol in reaction to the OFT (Kirschbaum and Hellhammer, 2000). Measuring salivary cortisol is preferred over blood sampling, since the method of sampling is much less aversive (Mormède et al., 2007). The piglet was allowed to chew on two cotton swabs (Cotton Swabs $150 \times 4 \mathrm{~mm}$ WA 2PL, Heinz Herenz, Hamburg, Germany) until they were thoroughly moistened with saliva. The swabs were then immediately stored in tubes on ice. After the samples were centrifuged at $2200-3000 \mathrm{~g}$ for $10-15 \mathrm{~min}$ at room temperature, the saliva was pipetted in $2 \mathrm{ml}$ Eppendorf tubes and stored at $-20^{\circ} \mathrm{C}$ (as adapted from Merlot et al., 2011). All samples were assayed on the same day in duplicate by radioimmunoassayusing methods, adapted from the manufacturer's procedure (Coat-a-Count cortisol TKCO, Siemens Solution Diagnostics, Los Angeles, CA, USA).

Baseline cortisol levels could be established for all pigs. The samples of three pigs taken after the open field test did not contain sufficient saliva for analysis.

\subsection{Holeboard test}

\subsubsection{The holeboard apparatus}

The holeboard apparatus - built by Ossendrijver B.V., Achterveld, The Netherlands - was located next to the home pens. The apparatus measured $530 \times 530 \mathrm{~cm}$, with synthetic grey walls that were $80 \mathrm{~cm}$ high, and a blue slatted synthetic floor that was elevated $25 \mathrm{~cm}$ from the ground. The arena was surrounded by a $40 \mathrm{~cm}$ wide corridor, which contained the main entrance to the apparatus. Via a guillotine door in the centre of each side wall, operated manually by the experimenter using a rope and pulley system, the pig could enter the holeboard arena. The arena contained a four by four matrix of 'holes' where the pig could search for food rewards (for details see Fig. 1 in Antonides et al., 2015). A hole consisted of a plastic food bowl with a false bottom (Road Refresher Large, Prestige Pet Products, Essex, England), covered by a red plastic ball (JollyBall Dog Toy, ø $24 \mathrm{~cm}, 1400$ g, Jolly Pets, Ohio, USA) to prevent the pig from locating the rewards using visual cues.

The pig had to lift the ball using its snout to uncover the food bowl and consume the reward. Chocolate M\&M's ${ }^{\circledR}$ were placed as rewards on the false bottom of the bowl. Underneath the false bottom of all holes, 3 inaccessible M\&M's ${ }^{\circledR}$ were replaced daily to ensure that pigs could not locate rewards using olfactory cues. Whenever a ball was lifted from the food bowl, the connection between a sensor in the bowl and a magnet in the ball 

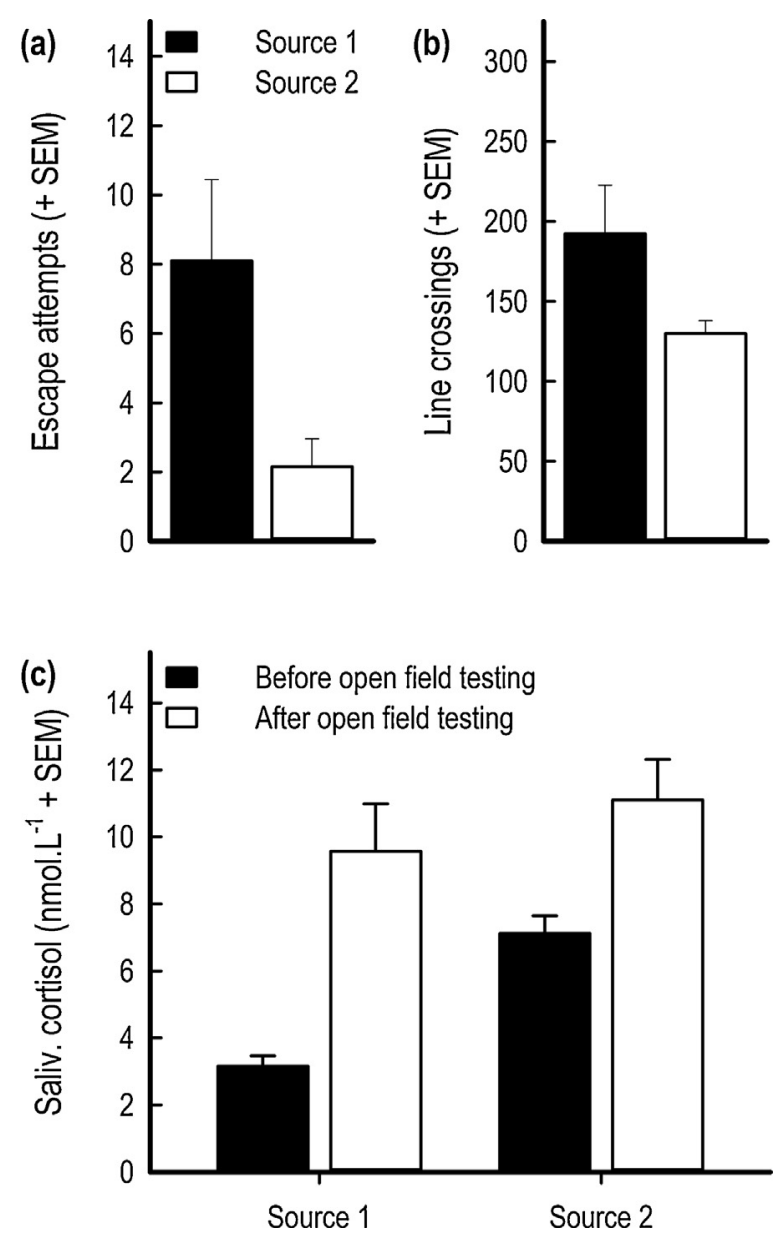

Fig. 2. Behaviours in the open field test, depicted by source. The test was conducted individually at the age of five weeks and lasted ten minutes. (a) Number of escape attempts; (b) Number of line crossings; (c) Salivary cortisol values before and after the open field testing, depicted by source.

was broken, which generated a signal that was registered by an interface (LabJack), connected to a laptop. This signal was processed by a tailor-made software program (Blinq Systems, Delft, The Netherlands). A steel wire attached to the top of the ball ensured that the ball always fell back into place with the magnet located directly above the sensor. Lifting a ball multiple times during a 10 -second-period, without lifting another ball, was recorded as one visit.

\subsubsection{Holeboard testing procedure}

After the open field test at 5 weeks of age, piglets were habituated to the waiting area and the holeboard apparatus for approximately 15 min per day for 4 weeks. The habituation period lasted until all piglets were comfortable staying in the holeboard arena alone, lifted the balls and consumed rewards, which was at approximately 9 weeks of age. From age 9-14 weeks, the piglets were tested in the holeboard task in three successive phases: the habituation phase ( 6 trials), the acquisition phase ( 40 trials) and the reversal phase (40 trials). Pigs that had reached the pre-set criterion of an average reference memory score $\geq 0.7$ (see Section 2.8.4 for a definition of this measure) at the end of the acquisition phase, over the last trial block (trials 37-40), were switched to the reversal phase. If a pig did not meet the criterion after 40 acquisition trials, its acquisition phase was extended until it did, with a maximum of 60 trials. If a pig did not reach the criterion within 60 acquisition trials, it was excluded from testing in the reversal phase. This was the case for one small litter piglet from Source 1.
During the habituation trials, all 16 holes were baited with M\&M's ${ }^{\circledR}$ chocolates. In the acquisition phase, each pig was assigned its own configuration of four baited holes. In total, four different configurations were used, in such a way that, across pigs, every hole was baited equally often (see Fig. 2, second panel in Antonides et al., 2015). The reversal configuration was the $180^{\circ}$ rotated pattern of baited holes used during the acquisition phase.

For each trial, the entry door was randomly determined by the software that controlled holeboard testing and collected the data. A trial was started when the pig entered the arena with its two front legs, and ended automatically when all rewards had been found, or when the maximum trial duration of $450 \mathrm{~s}$ had elapsed, whichever event occurred first. Piglets always received two trials in close succession. The pen order in which the piglets were tested was alternated each testing day. A pig inside the holeboard could smell and hear its pen mates in the waiting pen next to the holeboard apparatus. Fluorescent lights on the sloping ceiling of the stable and the position of the experimenter standing in the corridor of the holeboard arena served as visual extra-maze cues for spatial orientation.

\subsection{Statistical analyses}

All data were analysed using SAS version 9.4 for Windows (SAS Institute, Cary, North Carolina, USA). Normal distribution of the residuals of all variables was assessed using the Shapiro-Wilk test (SAS PROC UNIVARIATE). All analyses of variance (ANOVA) were performed using SAS PROC GLM, except for the open field data which were analysed parametrically or as ranked scores (Friedman's Two-way Nonparametric ANOVA for ranked data (Friedman, 1937) using PROC ANOVA. Where appropriate, we performed Sidak post-hoc comparisons between the four groups of piglets.

\subsubsection{Birth weight and growth}

Birth weights of the piglets were analysed by a Litter size by Source ANOVA. Growth from 4 to 15 weeks of age were analysed using an ANOVA with the repeated measures factor Week, and with Litter size and Source as between-subjects factors, and their interactions.

\subsubsection{Open field data}

Intra- and inter-rater reliabilites were determined using a Spearman's rank correlations test (as described in Martin and Bateson, 1993). Intra-rater reliability was determined for the variables number of escape attempts, number of times the piglet looked at the novel object, and number of times the piglet touched the novel object. These variables were scored twice by the same observer: directly during OFT and again from the video recordings. The number of vocalizations, number of line crossings and percentage of time spent in centre or wall segments were scored independently by two observers from the video recordings. Each observer scored $50 \%$ of all video recordings. Additionally, an overlap of $15 \%$ of all video recordings was scored by both observers to determine inter-rater reliability. For subsequent analyses, the scores of the two observations (live and from recordings by the same observer, or from the recordings by two observers) were averaged.

As there were eight outer segments and one centre segment, the mean percentage of time a piglet spent in an outer segment (i.e. near the walls and in the corners) was determined. The average time spent per outer segment was compared with time spent in the centre segment by adding segment (centre or outer) as repeated measures factor to determine whether piglets showed wall hugging behaviour (thigmotaxis) indicated by spending, on average, more time in an outer than in the centre segment. 

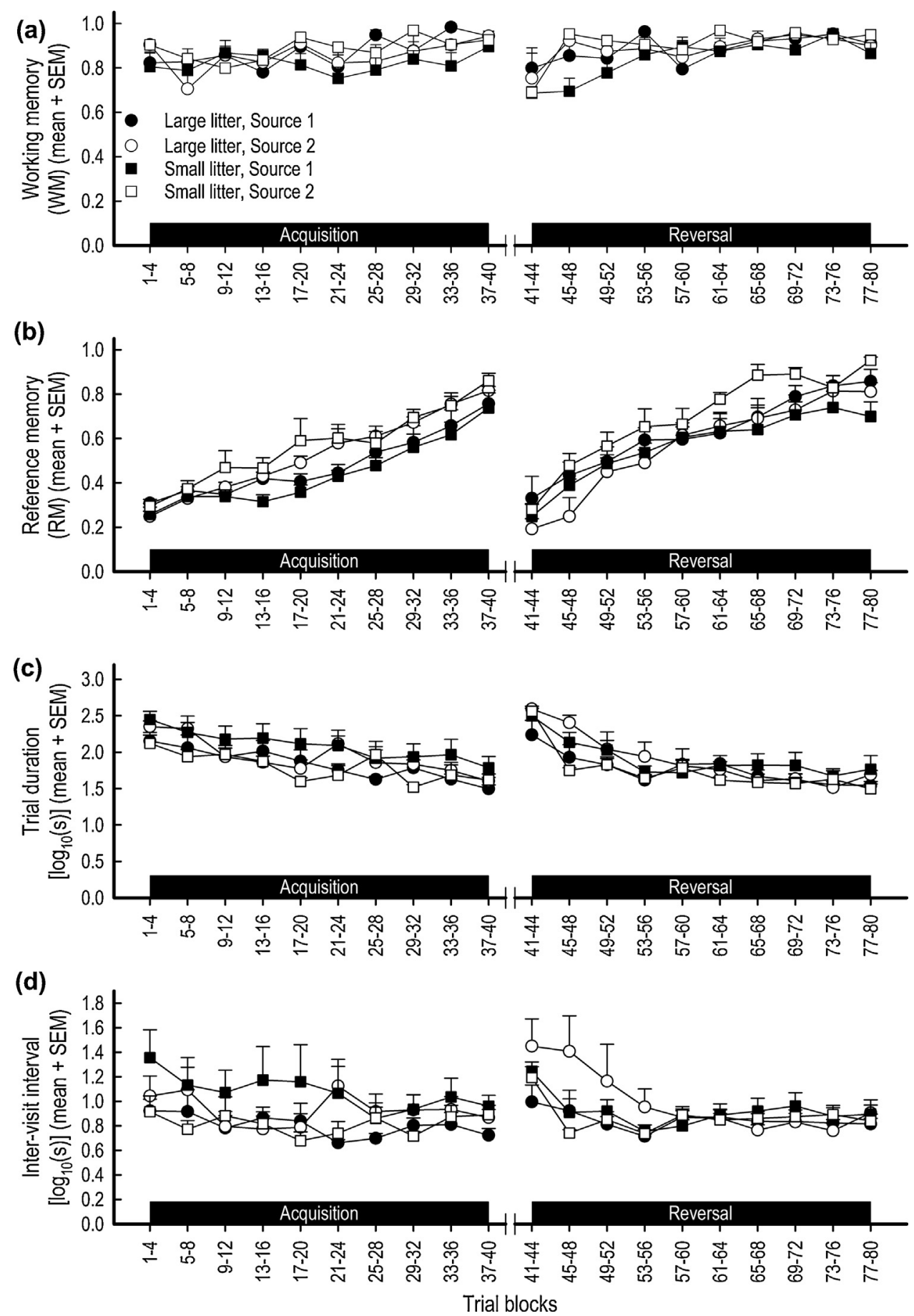

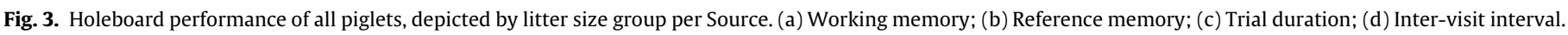

All other OFT variables were rank-transformed because they did not meet the normality assumption, and were analysed using the Friedman's Two-way Nonparametric ANOVA for ranked data (Friedman, 1937).

\subsubsection{Salivary cortisol data}

Baseline salivary cortisol values, cortisol values after the OFT, the absolute increase in cortisol and the proportional increase in cortisol with respect to baseline levels, were analysed with a two factorial ANOVA with the factors Litter size and Source, and their interaction.

\subsubsection{Holeboard data}

In the six habituation trials preceding actual holeboard testing, the total number of visits (TV) and number of unique hole visits (UHV) were recorded per trial. Note that UHV corresponds to the number of rewards that were found, as all holes were baited during this phase. TV and UHV scores were analysed using a repeated measures ANOVA with Litter size and Source as between-subjects factors and Trials as within-subject factor.

During the acquisition and reversal phase, the following variables were recorded or calculated (van der Staay et al., 2012): Working memory (WM): a ratio defined by the number of visits that yield a food reward divided by the number of visits and re-visits to 
the rewarded set of holes; Reference memory (RM): a ratio that is defined by the number of visits and re-visits to the rewarded set of holes divided by the number of visits and re-visits to all holes; Trial duration (TD): the time (s) between entering the holeboard and finding all four rewards (when not all rewards were found, the maximum trial duration of $450 \mathrm{~s}$ was recorded); and the Inter-visit interval (IVI): the average time (s) between two hole visits.

For all holeboard variables measured in the acquisition and reversal phase, means of 4 successive trials (trial block means) were calculated. Trial block means of variables expressing latencies or durations (TD and IVI) were $\log _{10}$-transformed to meet the normality assumption. Analyses were performed for the acquisition phase (40 trials; blocks 1-10), the reversal phase (40 trials; blocks 11-20) and the transition phase. The transition phase is the switch from the acquisition phase to the reversal phase, i.e. the last trial block mean of the acquisition phase compared to the first trial block mean of the reversal phase (block 10 compared to block 11). This is a measure of the response flexibility of an animal: a large difference may indicate that the animal faced difficulties to adapt to the new configuration of baited holes. Alternatively, it may imply that piglets had learned the assigned configuration in the acquisition phase very well. The extra acquisition trials that a piglet received when it had not yet reached the pre-set criterion of $\mathrm{RM} \geq 0.7$ after 40 trials were excluded from analyses.

Holeboard data were analysed using a repeated measures ANOVA with Litter size and Source as between-subjects factors, Trial blocks as within-subject factor, and their interactions.

\section{Results}

\subsection{Birth weights and growth}

Piglets from large litters had lower average birth weights $(1.31 \pm 0.05 \mathrm{~kg})$ than piglets from small litters $(1.75 \pm 0.10 \mathrm{~kg})$ (Litter size: $F_{1,16}=12.40, P=0.003$ ) (Fig. $1 \mathrm{~A}$ ). There was no difference in birth weight between piglets from the two different sources (Source: $F_{1,16}=0.00, P=0.98$; Litter size by Source interaction: $F_{1,16}=0.30, P=0.59$ ).

Source and litter size differentially affected the average body weight (Litter size by Source interaction: $F_{1,16}=7.75, P=0.01$ ) and the growth rate across weeks $4-15$ (Week by Litter size by Source interaction: $F_{11,176}=2.70, P=0.003$ ). Sidak post-hoc comparisons between the four groups of piglets revealed that body weight of the piglets from small litters of Source 1 exceeded that of the other three groups of piglets, starting in week 6 (Fig. 1B). The body weights of the other three groups of piglets did not differ from one another.

\subsection{Open field test}

\subsubsection{Behaviours in the open field test}

Reliabilities: The intra- and inter-rater reliabilities for the OFT observations were $\geq 0.73$ and $\geq 0.78$, respectively.

Piglets from Source 1 made more escape attempts (Source: $F_{1,16}=5.57, P=0.03 ;$ Fig. $2 \mathrm{~A}$ ) and line crossings (Source: $F_{1,16}=4.81$, $P=0.04$; Fig. $2 B$ ) than piglets from Source 2. Piglets from Source 2 tended to vocalize more during the OFT than piglets from Source 1 (Source: $F_{1,16}=3.48, P=0.08$ ). Litter size had no effect on any of these three variables.

The piglets spent a higher percentage of time in the centre segment than on average, in each of the eight outer segments (Segment: $F_{1,16}=12.72, P=0.003$ ), i.e. they showed no thigmotaxis in the OFT. Litter size or Source did not affect the percentage of time spent in centre or outer segments.

\subsection{Cortisol}

Sampling of saliva after the OFT yielded insufficient amounts for cortisol determinations in one small litter piglet from Source 1 and two small piglets from Source 2 .

Piglets from Source 2 had higher baseline salivary cortisol values than piglets from Source 1 (Source: $F_{1,13}=4.85, P=0.046$; Fig. $2 \mathrm{C}$ ). All groups showed increased levels of salivary cortisol after the $\operatorname{OFT}\left(F_{1,13}=36.60, P<0.0001\right)$. The absolute increase was similar for suppliers and litter sizes.

However, piglets from Source 1 showed a higher proportional increase in salivary cortisol after the OFT $(295 \pm 31 \%)$ than piglets from Source $2(151 \pm 11 \%)$ (Source: $\left.F_{1,13}=15.86, P=0.002\right)$.

\subsection{Holeboard task performance}

Table 1 shows a summary of the effects of source and the differential effects of litter size per source on holeboard performance of the piglets.

\subsubsection{Habituation trials}

In the six habituation trials preceding actual testing, no effects of litter size or source on the total number of visits (TV) or unique hole visits (UHV) were found. The four groups of piglets showed a constant number of visits (Trials: $F_{5,80}=0.41, P=0.84$ ), but a change in UHV over the six habituation trials (Trials: $F_{5,80}=2.88, P=0.02$ ). However, the change of UHV was not a systematic increase as might be expected, but an unsystematic fluctuation across the six trials.

The average UHV for all animals over the six trials was $14 \pm 0.4$ unique hole visits. UHV in this phase corresponds to the number of rewards found, as all 16 holes were baited. Therefore, this average UHV of 14 shows that the animals effectively searched throughout the holeboard for rewards before actual testing started.

\subsubsection{Working memory}

Working memory (WM; see Fig. 3A) performance slightly improved for all animals during the acquisition phase (Trial blocks: $F_{9,144}=3.96, P=0.0002$ ) and the reversal phase (Trial blocks: $\left.F_{9,135}=7.07, P<0.0001\right)$, and decreased for all piglets in the transition phase (Trial blocks: $F_{1,16}=18.05, P=0.0006$; Fig. $3 \mathrm{~A}$ ).

Neither Litter size nor Source affected WM performance during the acquisition phase. In the reversal phase, piglets from Source 2 showed higher average WM scores than piglets from Source 1 (Source: $F_{1,15}=5.34, P=0.04$ ). Of the four groups of pigs, small litter piglets from Source 1 had, on average, the lowest WM scores in the reversal phase (Litter size by Source interaction: $F_{1,15}=4.64$, $P=0.048$ ).

\subsubsection{Reference memory}

Reference memory (RM; see Fig. 3B) performance during both the acquisition (Trial blocks: $F_{9,144}=81.42, P<0.0001$ ) and reversal phase (Trial blocks: $F_{9,144}=51.04, P<0.0001$ ) increased in the four groups. They showed a similar drop in RM scores in the transition phase (Trial blocks: $F_{1,16}=108.59, P<0.0001$ ). During the acquisition phase, piglets from Source 2 showed faster RM learning (Trial blocks by Source interaction: $F_{9,144}=2.44, P=0.01$ ) than piglets from Source 1 (Fig. 3B).

RM performance of large litter piglets from Source 1 appeared to be least affected by the transition (Litter size by Source interaction: $\left.F_{1,16}=5.70, P=0.03\right)$. During the reversal phase, small litter piglets from Source 2 outperformed the other three groups on the average RM performance (Litter size by Source interaction: $F_{1,16}=7.51$, $P=0.01)$. 
Table 1

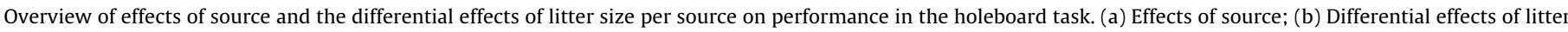
size per source.

(a) Effect of source on holeboard performance

\begin{tabular}{|c|c|c|c|}
\hline Phase & Measure & Difference found between sources & See figure \\
\hline \multirow[t]{3}{*}{ Acquisition } & Reference memory (RM) & Source 2: Piglets show faster increase in RM & Fig. 3B \\
\hline & Trial duration (TD) & Different slopes in TD between piglets of the two sources & Fig. 3C \\
\hline & Inter-visit interval (IVI) & Different slopes in IVI between piglets of the two sources & Fig. 3D \\
\hline Reversal & Working memory (WM) & Source 2: Piglets show better WM performance & Fig. 3A \\
\hline
\end{tabular}

(b) Differential effect of litter size on holeboard performance per source

\begin{tabular}{|c|c|c|c|c|}
\hline Phase & Measure & Effect in pigs of & Litter size group & See figure \\
\hline \multirow[t]{2}{*}{ Transition } & Reference memory (RM) & Source 1 & Large litters: Smaller drop in RM score & Fig. 3B \\
\hline & Trial duration (TD) & Source 1 & Large litters: Smaller rise in TD score & Fig. 3C \\
\hline \multirow[t]{2}{*}{ Reversal } & Reference memory (RM) & Source 2 & Small litters: Higher RM scores, i.e. better performance & Fig. 3B \\
\hline & Inter-visit interval (IVI) & Source 2 & Different slope between litter size groups & Fig. 3D \\
\hline
\end{tabular}

\subsubsection{Trial duration}

The Trial duration (TD; see Fig. 3C) decreased in both the acquisition (Trial blocks: $F_{9,144}=22.49, P<0.0001$ ) and the reversal phase (Trial blocks: $F_{9,144}=31.09, P<0.0001$ ), and increased in the transition phase for all groups (Trial blocks: $F_{1,16}=99.67, P<0.0001$ ).

During the acquisition phase, the piglets from Source 1 showed a different change in TD across trial blocks than piglets from Source 2 (Trial blocks by Source interaction: $F_{9,144}=2.44, P=0.01$ ). The average TD during the transition was different for piglets from small and large litters from the two sources (Litter Size by Source interaction: $\left.F_{1,16}=4.76, P=0.04\right)$. Piglets from large litters of Source 1 showed the smallest increase in TD during the transition.

\subsubsection{Inter-visit interval}

The Inter-visit interval (IVI; see Fig. 3D) slightly declined during the acquisition (Trial blocks: $F_{9,144}=3.38, P=0.0009$ ) and reversal phase (Trial blocks: $F_{9,144}=10.76, P<0.0001$ ), and increased during the transition phase for all animals (Trial blocks: $F_{1,16}=37.25$, $P<0.0001$ ).

During the acquisition phase, piglets from Source 1 showed a different change in IVI than piglets from Source 2 (Trial blocks by Source interaction: $F_{9,144}=2.78, P=0.005$ ). Large litter piglets from Source 2 tended to show a steeper decrease of IVI in the reversal phase than the other three groups (Trial blocks by Source by Litter size interaction: $\left.F_{9,144}=1.71, P=0.093\right)$.

\section{Discussion}

The aim of the present study was to investigate whether litter size affects emotionality, spatial learning and memory in piglets. We expected that piglets from large litters would display higher emotionality during the open field test (OFT), show a greater surge in cortisol after the OFT, and perform worse in the holeboard task than piglets from relatively small litters.

\subsection{Supplier or breed?}

Due to technical problems, the design of the study changed from a simple two-groups comparison (small vs. large litter) to a factorial design with as extra factor Source (supplier/breed). In this factorial design, the effects of litter size are determined by comparing all piglets from small litters with all piglets from large litters, i.e. this main effect is tested with the same number of animals for both levels of the factor "litter size" as in the original setup (see also Shaw et al., 2002). If this effect is robust, then Source should not be relevant. At the same time, this design enables to assess the effects of the additional factor Source and its interaction with Litter size.
According to Festing et al. “(...) factorial experimental designs (...), in which two or more factors (for example, treatments, time, sex, strain, age, or diet) are varied simultaneously, usually make more efficient use of resources (including experimental animals) than do designs involving only a single factor (...)." (Festing et al., 1998). In the present design, testing the effects of Litter size can be considered as the confirmatory component, whereas the effects of Source and its interaction with Litter size can be considered as an exploratory component of the study. Experiments with a confirmatory and an exploratory component are not unusual (Festing and Altman, 2002).

This change in design thus does not affect precision (Festing, 1992; Shaw et al., 2002). It does add a second question, namely whether this effect (if present) is robust, i.e. whether or not it is affected by Source (supplier/breed).

We do not know whether the interaction effects between Source and Litter size found on growth rates and several behavioural measures in the open field and the holeboard task point to differential effect of either supplier or breed. These are, however, interesting new findings that raise questions worthy of future investigations.

Surprisingly, Source had a profound effect on activity and cortisol increase during the OFT and some effects on memory performance in the holeboard task. Moreover, interactions between Litter size and Source were found. These interaction effects imply that litter size has differential effects on growth and cognitive performance in piglets of different sources (i.e. suppliers and/or breeds).

Both suppliers provided roughly the same environmental conditions in terms of thermal regulation, feed and enrichment. However, they provided different crossbreeds of pigs. It is not possible to disentangle, in case of interaction effects, whether these effects are due to genetics of the piglets or to different management practices. Rather, genetics, management and/or environmental factors may have influenced development and behaviour in our pigs differently.

\subsection{Growth}

The birth weights of piglets from large litters were lower than those of piglets from small litters, which is in line with earlier studies (Bergstrom et al., 2009; Beaulieu et al., 2010). Surprisingly, small litter piglets from Source 1 grew faster than the other three groups over the course of the experiment. This implies that litter size had an effect on growth in piglets of Source 1, but not in piglets of Source 2. It also shows that the growth of piglets from Source 2 was more homogeneous than that of piglets from Source 1.

Source 2 supplied piglets bred and reared under SPF conditions. The growth of pigs from this source may therefore be less affected 
by differences in litter sizes and birth weight. However, small litter pigs from Source 1 showed faster growth and thus had higher body weights than large litter piglets from the same source at the end of the experiment. In the pig industry, high body weights are preferable as heavy pigs yield more meat.

\subsection{Open field test}

No effects of litter size were found on behavioural measures or cortisol increase in the OFT. Piglets from Source 1 showed higher locomotor activity and tended to vocalize less during the OFT than piglets from Source 2. Piglets in an OFT study treated with a stress reducing drug, and piglets accompanied by another piglet, showed more locomotion and less vocalization than controls (Donald et al., 2011). This would imply that piglets from Source 2 showed higher emotionality in the OFT.

In a study comparing active and passive behavioural response piglets in the OFT, active piglets were found to vocalize less, make more escape attempts, have lower baseline cortisol levels and show a greater increase in cortisol after the OFT than passive piglets (Hessing et al., 1994). Pigs from Source 1 in the present study tended to vocalize less, made more escape attempts, had higher baseline cortisol values and a greater proportional increase in cortisol after the OFT than pigs from Source 2. Thus, when comparing our results to those of the study of Hessing et al. (1994), Source 1 seems to supply active behavioural response pigs, and Source 2 passive behavioural response pigs. It should be noted that the difference in proportional increase in cortisol may be due to a ceiling effect, i.e. that all piglets reached a maximum value of cortisol after the OFT.

\subsection{Pigs show no wall hugging behaviour (thigmotaxis)}

It has been reported that pigs show a tendency to spend more time near the walls than in the centre segment of an open field (Donald et al., 2011). Forkman et al. (2007) argue that farm animals evolved in open areas, and thus may not show thigmotactic behaviour. We found that piglets spent relatively more time in the centre segment than in outer segments. This implies that piglets do not show thigmotactic behaviour, which is commonly found in rodents (Walsh and Cummins, 1976). In a review, Murphy et al. (2014) also conclude that it is unlikely that pigs show thigmotactic behaviour in the OFT. Our results imply that wall hugging behaviour in an OFT is not a useful measure to assess emotionality in young piglets.

In summary, we found strong effects of source on locomotor behaviour and cortisol measures during the OFT, yet no effects of litter size. We therefore argue that either supplier or breed has profound effects on emotionality responses in pigs, and that pigs from different suppliers or breeds can express different combinations of response behaviours when measuring emotionality.

\subsection{Holeboard task}

All piglets acquired the holeboard task and improved their performance during both the acquisition and the reversal phase, and were affected by the transition to another set of baited holes from the acquisition to the reversal phase. This corroborates earlier findings of studies using the holeboard task in pigs (Arts et al., 2009; Gieling et al., 2012; Antonides et al., 2015).

\subsubsection{Strong effects of source on holeboard performance}

Piglets from Source 2 showed faster reference memory (RM) learning in the acquisition phase and different changes in trial duration (TD) and inter-visit interval (IVI) than piglets from Source 1.
In the reversal phase, piglets from Source 2 had higher working memory (WM) scores than piglets from Source 1.

Taken together, piglets from Source 2 partially outperformed piglets from Source 1 in the holeboard task, showing faster RM learning in the acquisition phase and higher WM scores in the reversal phase of the task.

\subsubsection{Effects of litter size on holeboard performance: different per source}

Litter size had a differential effect on holeboard performance of the piglets purchased from the two different sources. In the acquisition phase, no effects of litter size on holeboard performance were found. In the transition phase, large litter piglets from Source 1 showed a smaller drop in RM performance and a smaller rise in TD than small litter piglets of the same source. This may indicate that large litter piglets from Source 1 were more flexible in re-learning where to find rewards than small litter piglets. Alternatively, this may imply that they had a weaker consolidation of the original configuration of baited holes than small litter piglets.

In the reversal phase, litter size had an effect on RM performance only in piglets of Source 2: small litter piglets showed higher overall RM scores than large litter piglets. Additionally, an effect of Litter size in piglets from Source 2 was found on the changes in IVI during the reversal phase. Although these changes are nonlinear and it is thus difficult to interpret this result, it does show that there were some litter size effects within pigs of Source 2 that were not found in pigs of Source 1.

\subsection{Critical notes}

It is possible that the difference in litter size between small $(\leq 13$ piglets) and large litters ( $\geq 18$ piglets) in our study was not large enough to detect effects of litter size. It may, therefore, be advisable to increase the difference in litter size between groups in future studies. However, this may prove to be challenging, as small litters are expected to become increasingly infrequent in the pig industry (Rutherford et al., 2013).

The set-up of our study included piglets from two different suppliers, supplying different breeds. If litter size would have a strong and robust effect on performance in behavioural tasks, we would expect that litter size effects are robust over different breeds of pigs and (thus) over pigs from different suppliers. However, as we included both litter size and source as factors in our analyses, interaction effects were estimated based on 5 piglets per group. It is plausible that this has reduced the power of our analyses for interaction effects. Although we found indications that Source and Litter size had a differential effect on several behaviours, we recommend that this is further investigated using more animals.

\subsection{Conclusion}

Taken together, our results show that Source (i.e. supplier and/or breed) can have a strong effect on performance in behavioural tasks, and that effects such as Litter size may affect pigs' performance of one source, while it may not or differently affect pigs from another source. These findings show that it is important to realise that results from behavioural tasks may not be generalizable across different breeds of pigs or across pigs from different suppliers.

\section{References}

Antonides, A., Schoonderwoerd, A.C., Nordquist, R.E., van der Staay, F.J., 2015. Very low birth weight piglets show improved cognitive performance in the spatial cognitive holeboard task. Front. Behav. Neurosci., 9.

Arcangeli, T., Thilaganathan, B., Hooper, R., Khan, K.S., Bhide, A., 2012. Neurodevelopmental delay in small babies at term: a systematic review. 
Ultrasound Obstet. Gynecol. Off. J. Int. Soc. Ultrasound Obstet. Gynecol. 40 267-275, http://dx.doi.org/10.1002/uog.11112.

Arts, J.W.M., van der Staay, F.J., Ekkel, E.D., 2009. Working and reference memory of pigs in the spatial holeboard discrimination task. Behav. Brain Res. 205 303-306, http://dx.doi.org/10.1016/j.bbr.2009.06.014

Beaulieu, A.D., Aalhus, J.L., Williams, N.H., Patience, J.F., 2010. Impact of piglet birth weight, birth order, and litter size on subsequent growth performance, carcass quality, muscle composition, and eating quality of pork. J. Anim. Sci. 88 2767-2778, http://dx.doi.org/10.2527/jas.2009-2222.

Bergstrom, J.R., Potter, M.L., Henry, S.C., Tokach, M.D., Nelssen, J.L., Goodband, R.D. DeRouchey, J.M., Dritz, S.S., 2009. Effects of Piglet Birth Weight and Litter Size on the Preweaning Growth Performance of Pigs on a Commercial Farm. Kansas State University. Agricultural Experiment Station and Cooperative Extension Service, pp. 1-7 (Presented at the Swine Day, Manhattan, KS, November 19, 2009).

Donald, R.D., Healy, S.D., Lawrence, A.B., Rutherford, K.M.D., 2011. Emotionality in growing pigs: is the open field a valid test? Physiol. Behav. 104, 906-913, http://dx.doi.org/10.1016/j.physbeh.2011.05.031.

Festing, M.F.W., Altman, D.G., 2002. Guidelines for the design and statistical analysis of experiments using laboratory animals. ILAR J. 43, 244-258, http:// dx.doi.org/10.1093/ilar.43.4.244.

Festing, M.F.W., Baumans, V., Combes, R.D., Halder, M., Hendriksen, C.F.M. Howard, B.R., Lovell, D.P., Moore, G.M., Overend, P., Wilson, M.S., 1998. Reducing the use of laboratory animals in biomedical research: problems and possible solutions-The report and recommendations of ECVAM workshop 291,2,3. Altern. Lab. Anim. ATLA 26, 283-301.

Festing, M.F.W., 1992. The scope for improving the design of laboratory animal experiments. Lab. Anim. 26, 256-267, http://dx.doi.org/10.1258/ 002367792780745788.

Forkman, B., Boissy, A., Meunier-Salaün, M.-C., Canali, E., Jones, R.B., 2007. A critical review of fear tests used on cattle, pigs, sheep, poultry and horses. Physiol. Behav. Stress Welf. Farm Anim. 92, 340-374, http://dx.doi.org/10.1016/j. physbeh.2007.03.016.

Friedman, M., 1937. The use of ranks to avoid the assumption of normality implicit in the analysis of variance. J. Am. Stat. Assoc. 32, 675-701.

Gieling, E.T., Park, S.Y., Nordquist, R.E., van der Staay, F.J., 2012. Cognitive performance of low-and normal-birth-weight piglets in a spatial hole-board discrimination task. Pediatr. Res. 71, 71

Herpin, P., Damon, M., Le Dividich, J., 2002. Development of thermoregulation and neonatal survival in pigs. Livest. Prod. Sci. Peri- Post-Natal Mortal. Pig 78, 25-45, http://dx.doi.org/10.1016/S0301-6226(02) 00183-5.

Hessing, M.J.C., Hagelsø, A.M., Schouten, W.G.P., Wiepkema, P.R., Van Beek, J.A.M., 1994. Individual behavioral and physiological strategies in pigs. Physiol. Behav. 55, 39-46, http://dx.doi.org/10.1016/0031-9384(94)90007-8.

Kirschbaum, C., Hellhammer, D.H., 2000. Salivary cortisol. Encycl. Stress, 3.

Larroque, B., Bertrais, S., Czernichow, P., Léger, J., 2001. School difficulties in 20 -year-olds who were born small for gestational age at term in a regional cohort study. Pediatrics 108, 111-115, http://dx.doi.org/10.1542/peds.108.1 111.

Mainau, E., Dalmau, A., Ruiz-de-la-Torre, J.L., Manteca, X., 2010. A behavioural scale to measure ease of farrowing in sows. Theriogenology 74, 1279-1287, http:// dx.doi.org/10.1016/j.theriogenology.2010.05.034.

Martin, P., Bateson, P., 1993. Measuring Behavior: An Introductory Guide, 2nd ed. Cambridge University Press, Cambridge, UK.
Merlot, E., Mounier, A.M., Prunier, A., 2011. Endocrine response of gilts to various common stressors: a comparison of indicators and methods of analysis. Physiol. Behav. 102, 259-265, http://dx.doi.org/10.1016/j.physbeh.2010.11. 009.

Mormède, P., Andanson, S., Aupérin, B., Beerda, B., Guémené, D., Malmkvist, J., Manteca, X., Manteuffel, G., Prunet, P., van Reenen, C.G., et al., 2007. Exploration of the hypothalamic-pituitary-adrenal function as a tool to evaluate animal welfare. Physiol. Behav. 92, 317-339.

Murphy, E., Nordquist, R.E., van der Staay, F.J., 2014. A review of behavioural methods to study emotion and mood in pigs, Sus scrofa. Appl. Anim. Behav. Sci. 159, 9-28.

Norring, M., Valros, A., Munksgaard, L., Puumala, M., Kaustell, K.O., Saloniemi, H., 2006. The development of skin, claw and teat lesions in sows and piglets in farrowing crates with two concrete flooring materials. Acta Agric. Scand. Sect. - Anim. Sci. 56, 148-154, http://dx.doi.org/10.1080/09064700701285065.

Ogata, E.S., Bussey, M.E., Labarbera, A., Finley, S., 1985. Altered growth, hypoglycemia hypoalaninemia, and ketonemia in the young rat: postnatal consequences of intrauterine growth retardation. Pediatr. Res. 19, 32-37, http://dx.doi.org/10.1203/00006450-198501000-00010.

Père, M.-C., Etienne, M., 2000. Uterine blood flow in sows: effects of pregnancy stage and litter size. Reprod. Nutr. Dev. 40, 369-382.

Prunier, A., Heinonen, M., Quesnel, H., 2010. High physiological demands in intensively raised pigs: impact on health and welfare. Animal 4, 886-898 http://dx.doi.org/10.1017/S175173111000008X.

Quiniou, N., Dagorn, J., Gaudré, D., 2002. Variation of piglets birth weight and consequences on subsequent performance. Livest. Prod. Sci. Peri- Post-Natal Mortal. Pig 78, 63-70, http://dx.doi.org/10.1016/S0301-6226(02) 00181-1.

Rutherford, K.M., Baxter, E.M., Ask, B., Berg, P., DEath, R.B., Jarvis, S., Jensen, K.K., Lawrence, A.B., Moustsen, V.A., Robson, S.K. others, 2011. The ethical and welfare implications of large litter size in the domestic pig: challenges and solutions. Danish Cent. Bioeth. Risk Assess. (CeBRA).

Rutherford, K., Baxter, E., DEath, R., Turner, S., Arnott, G., Roehe, R., Ask, B., Sandøe, P., Moustsen, V., Thorup, F., Edwards, S., Berg, P., Lawrence, A., 2013. The welfare implications of large litter size in the domestic pig I: biological factors. Anim. Welf. 22, 199-218, http://dx.doi.org/10.7120/09627286.22.2.199.

Saito, A., Matsui, F., Hayashi, K., Watanabe, K., Ichinohashi, Y., Sato, Y., Hayakawa M., Kojima, S., Oohira, A., 2009. Behavioral abnormalities of fetal growth retardation model rats with reduced amounts of brain proteoglycans. Exp. Neurol. Brain Stimulation Psychiatry 219, 81-92, http://dx.doi.org/10.1016/j. expneurol.2009.04.012

Shaw, R., Festing, M.F.A., Peers, I., Furlong, L., 2002. Use of factorial designs to optimize animal experiments and reduce animal use. ILAR J. 43, 223-232.

Wahner, M., Fisher, K., 2005. Current physiological aspects of fetal growth and parturition in the pig. Biotechnol. Anim. Husb. 21, 135-148.

Walsh, R.N., Cummins, R.A., 1976. The open-field test: a critical review. Psychol. Bull. 83, 482-504, http://dx.doi.org/10.1037/0033-2909.83.3.482.

de Passillé, A.M.B., Rushen, J., 1989. Using early suckling behavior and weight gain to identify piglets at risk. Can. J. Anim. Sci. 69, 535-544, http://dx.doi.org/10. 4141/cjas89-066.

van der Staay, F.J., Gieling, E.T., Pinzón, N.E., Nordquist, R.E., Ohl, F., 2012. The appetitively motivated cognitive holeboard: a family of complex spatial discrimination tasks for assessing learning and memory. Neurosci. Biobehav. Rev. 36, 379-403, http://dx.doi.org/10.1016/j.neubiorev.2011.07.008. 